\title{
Schweizerische Gesellschaft für Bildungsforschung - Rückblenden auf Aspekte ihrer 40-jährigen Geschichte
}

\section{Silvia Grossenbacher}

Vor dem Hintergrund der institutionellen und forschungspolitischen Entwicklung im Bereich Bildungsforschung beleuchtet der Beitrag einige Aspekte der 40-jährigen Geschichte der Schweizerischen Gesellschaft für Bildungsforschung [SGBF]. Seit ihrer Gründung im Jahr 1975 bemühte sich die Gesellschaft durchaus selbstkritisch um die Weiterentwicklung dieses multidisziplinären Forschungsbereiches. In groben Zügen wird nachgezeichnet, was die Gesellschaft zur Profilierung des Forschungsbereiches und zur Unterstützung der Forschenden unternahm, welche forschungspolitischen Schwerpunkte sie verfolgte und wie sie Kompetenzaufbau und Nachwuchsförderung vorantrieb. Schliesslich wird auch das Diskussionsforum der Gesellschaft, die "Schweizerische Zeitschrift für Bildungswissenschaften", gewürdigt.

\section{Einle itung}

Der Beitrag wurde aus Anlass des 40-jährigen Bestehens der Schweizerischen Gesellschaft für Bildungsforschung $[S G B F]$ verfasst ${ }^{1}$. Er basiert nicht auf einer systematischen Aufarbeitung der Geschichte dieser wissenschaftlichen Gesellschaft, da diese Arbeit noch aussteht. Vielmehr handelt es sich um eine rekonstruktive Annäherung an einige Aspekte der Tätigkeit der SGBF².

Es liegt in der Natur der Sache, dass die Geschicke und die Leistungen der Schweizerischen Gesellschaft für Bildungsforschung nicht losgelöst von der institutionellen und forschungspolitischen Entwicklung dieses Wissenschaftsbereiches aufgezeigt werden können. Einleitend soll daher auf diese Entwicklung eingegangen werden, abgestützt auf verschiedene Bestandesaufnahmen ${ }^{3}$ der Bildungsforschung in der Schweiz, die in den letzten 40 Jahren vorgelegt worden sind (CORECHED, 2006; Gretler, 2000; Grossenbacher \& Gretler, 1992; Grossenbacher \& Oggenfuss, 2015a; Hofstetter \& Schneuwly, 2001; Patry \& Gretler, 1992; SGBF, 1988). 


\section{Bildungsforschung in der Schweiz - ein überblick}

Im Jahrzehnt zwischen 1965 und 1974 verzeichnete die Bildungsforschung in der Schweiz ein beträchtliches institutionelles Wachstum, sowohl innerhalb wie auch ausserhalb des universitären Rahmens. Während auf der Hochschulebene zum grössten Teil bereits bestehende Lehrstühle zu universitären Instituten ausgebaut wurden, handelt es sich bei den nicht-universitären Institutionen vorab um kantonale pädagogische Arbeitsstellen, die im Rahmen der Bildungsverwaltung Aufgaben angewandter Forschung und Entwicklung wahrnahmen (Bain, Brun, Hexel \& Weiss, 2001; SGBF, 1988). Die Expansion der verwaltungsinternen Dienste, die sehr unterschiedliche Kapazitäten aufwiesen und sich auch im Selbstverständnis hinsichtlich ihrer Forschungsaktivitäten unterschieden, wird zum einen auf den Reformdruck im Bildungswesen und zum andern auf Konflikte zwischen den kurzfristigen Bedürfnissen von Politik und Verwaltung und den eher längerfristigen wissenschaftlichen Interessen der Hochschulen zurückgeführt (Gretler, 2000; Hubermann, 1989). Auf Seite der Hochschulen befassten sich neben den Pädagogischen Instituten zunehmend auch andere Institutionen aus verschiedenen disziplinären Perspektiven mit Bildungsthemen. Der Begriff «Bildungsforschung» weist in diesem Sinne über die engere Disziplin Erziehungswissenschaft hinaus und definiert entsprechend ein breiteres multidisziplinäres Feld. Dieses wurde vom Schweizerischen Wissenschaftsrat zu Beginn der 1970er Jahre erstmals umschrieben und als ein prioritär zu förderndes Forschungsfeld eingestuft (SWR, 1970, 1973).

In der Periode 1975 bis 1990 setzte sich das institutionelle Wachstum fort, aber in bescheidenerem Ausmass. In dieser Konsolidierungsphase gewann die Bildungsforschung an Identität. Ein Ausdruck davon ist die Gründung der Schweizerischen Gesellschaft für Bildungsforschung im Jahre 1975 und die von der SGBF vier Jahre später lancierte Zeitschrift «Bildungsforschung und Bildungspraxis / Éducation et recherche». In den 1980er Jahren erstellte die Kommission Forschungspolitik der SGBF eine detaillierte Bestandesaufnahme der Bildungsforschung in der Schweiz. Sie zeigte neben Stärken der Bildungsforschung auch entscheidende Probleme. Dazu gehört der Mangel an kontinuierlicher und kumulativer Forschung, die institutionelle Fragmentierung in Klein- und Kleinstinstitutionen, die kaum die kritische Masse für eine langfristig angelegte und methodisch anspruchsvolle Forschung erreichten, oder auch eine chronische Unterfinanzierung. Aufgrund dieser Diagnose verabschiedete die SBGF den "Entwicklungsplan der schweizerischen Bildungsforschung» (SGBF, 1988). Eine vom Schweizerischen Wissenschaftsrat initiierte Untersuchung zur Situation der sozialwissenschaftlichen Forschung in der Schweiz (SOWI-Projekt), an der die Bildungsforschung als eine von vier Disziplinen teilnahm, bestätigte die im Entwicklungsplan identifizierten Probleme (Grossenbacher \& Gretler, 1992). Zur Stärkung der Bildungsforschung konnte 1991 auf Anregung der 
SGBF die Koordinationskonferenz Bildungsforschung CORECHED ins Leben gerufen werden. In dieser Konferenz wirkten die damaligen «Bildungsbundesämter» (Bundesamt für Bildung und Wissenschaft, Bundesamt für Industrie, Gewerbe und Arbeit), das Bundesamt für Statistik, die Erziehungsdirektorenkonferenz, der Nationalfonds und die SGBF zusammen mit dem Auftrag, die Entwicklung der Bildungsforschung laufend zu beobachten und den kontinuierlichen Austausch zwischen Forschung, Praxis, Politik und Verwaltung zu fördern (CORECHED, 1996).

Die Entwicklungsphase nach 1990 lässt sich mit den Begriffen Wirkungsorientierung und Umstrukturierung umschreiben. International gesehen richtete sich das Augenmerk von Politik und Forschung zunehmend auf Qualität, Wirkungen und Ergebnisse von Bildungsprozessen. Der Qualitätsdiskurs und der allgemeine Trend zur Rechenschaftslegung steigerten den Bedarf an wissenschaftlich fundierten Evaluationen von Programmen, Institutionen und Systemen. Während diese Aufgaben in der französisch- und italienischsprachigen Schweiz weitgehend von den verwaltungsinternen Forschungsdiensten übernommen wurden (Weiss, 2001), führte die Entwicklung in der Deutschschweiz u.a. zur Gründung privater Institutionen der Bildungsforschung. Zudem wurden im Rahmen des New Public Managements viele verwaltungsinterne Dienste in der Deutschschweiz reorganisiert oder aufgelöst und deren Forschungs- und Entwicklungsaufgaben teilweise in Form von Mandaten an die neu entstehenden Pädagogischen Hochschulen verlagert (Gretler, 2000).

Die jüngste Periode (ab 2000) ist gekennzeichnet durch institutionelle Differenzierung. Mit den Pädagogischen Hochschulen kamen um die Jahrtausendwende neue Spieler ins Feld und bauten auftragsgemäss ihre Forschungstätigkeit auf und aus (Grossenbacher \& Oggenfuss, 2015b). Der Bund reorganisierte seine Ressortforschung und investierte vermehrt in die Berufsbildungsforschung. Der Aufbau von Forschungsnetzwerken unter der Führung sogenannter Leading Houses führte zur besseren institutionellen Verankerung dieses Forschungszweiges (BBT, 2007). An den Deutschschweizer Universitäten wurden im erziehungswissenschaftlichen Bereich nach einer langen Phase mangelnden Ausbaus und lediglich moderater Differenzierung neue Lehrstühle geschaffen (Criblez, 2002). Die bisherigen Pädagogischen Institute erfuhren nach und nach eine Umbenennung in «Institute für Erziehungswissenschaft». Die Programme der OECD zu Bildungsindikatoren und Kompetenzmessungen beeinflussten auch Bildungsstatistik und Bildungsforschung in der Schweiz und schufen Grundlagen für den Aufbau von Bildungsmonitoring und -berichterstattung (Wolter, 2008). 


\section{Die Schweizerische Gesellschaft für Bildungs- forschung}

Im zweiten Teil des Artikels wird nun die Entwicklung der SGBF und ihrer Aktivitäten unter drei Gesichtspunkten beschrieben:

- Profilierung und Identitätsbildung, Förderung des Zusammenhalts, Unterstützung der Forschenden

- forschungspolitische Aktivitäten

- Kompetenzaufbau und Nachwuchsförderung.

\section{Gründungsphase}

Der Schweizerische Wissenschaftsrat hatte 1973 die Bildungsforschung zu einem der prioritär zu fördernden Forschungsbereiche erklärt, diese erstmals definitorisch umschrieben und die vorrangig zu bearbeitenden Forschungsfelder benannt (SWR, 1973). Neben "Umwelt» stellte der SWR «Bildung» in den Vordergrund der Sonderförderung, weil sie «für die künftige Entwicklung eines hochindustrialisierten Landes und für das Wohlergehen seiner Bevölkerung von grosser prospektiver Bedeutung sind» (ebd., S. 4). Vor diesem Hintergrund fand 1974 eine Fachtagung statt, an der unter anderem auf die Wünschbarkeit einer Gesellschaft für Bildungsforschung hingewiesen wurde. Ein Jahr später trafen sich in Bern 52 Personen, darunter 4 Frauen, um die Gesellschaft zu gründen (SGBF, Protokoll der Gründungsversammlung vom 28. Juni 1975). Die an der Gründungsversammlung diskutierten und verabschiedeten Statuten umrissen den Zweck grob als «Förderung der Bildungsforschung» und spezifizierten diesen wie folgt:

- «die in der Schweiz tätigen Bildungsforscher zusammenzuschliessen;

- die interdisziplinäre Zusammenarbeit zwischen ihnen zu fördern;

- die Zusammenarbeit mit andern Gesellschaften auf nationaler und internationaler Ebene zu pflegen;

- die Zusammenarbeit zwischen Bildungsforschung, -praxis, -verwaltung und -politik zu fördern;

- den Kontakt mit den wissenschaftspolitischen Instanzen zu pflegen;

- die Interessen der Bildungsforschung gegenüber wissenschaftspolitischen Instanzen, Bildungsverwaltung und -politik zu wahren;

- die Ergebnisse der Bildungsforschung zu verbreiten» (SGBF, Protokoll der Gründungsversammlung vom 28. Juni 1975, S. 3).

Ein Antrag auf Namensänderung in «Schweizerische Gesellschaft für Bildungswissenschaften» wurde abgelehnt. Als Mitglieder sollten der Gesellschaft Personen und Institutionen beitreten können, die in der Bildungsforschung tätig sind oder im Bildungswesen Funktionen in enger Zusammenarbeit mit Bildungsforschung wahrnehmen. Als erster Präsident wurde Marcel Goldschmid, Professor und Vorsteher des Departements Psychologie an der EPF in Lausanne, 
gewählt. Die an der Gründungsversammlung umrissenen Erwartungen an die Gesellschaft klingen anspruchsvoll. Im Protokoll werden u.a. folgende Punkte genannt:

- Kontakte mit wissenschaftspolitischen Instanzen

- Entwicklungsplan der schweizerischen Bildungsforschung

- Bemühen um ein Nationales Programm Bildungsforschung

- Gründung einer schweizerischen Zeitschrift für Bildungsforschung

- Durchführung einer grösseren Veranstaltung 1976 (SBGF, Protokoll der Gründungsversammlung vom 28. Juni 1975, S. 10).

Der Vorstand nahm seine Arbeit unverzüglich auf. Die Kontakte zu wissenschaftspolitischen Instanzen wurden geknüpft (Bundesämter, Schweizerischer Nationalfonds, Wissenschaftsrat, Erziehungsdirektorenkonferenz und Schweizerische Gesellschaft der Geisteswissenschaften [Vorläufer der Schweizerischen Akademie der Geistes- und Sozialwissenschaften SAGW]). Man rief Kommissionen und Arbeitsgruppen ins Leben und traf Vorbereitungen für einen ersten Kongress, der im Juni 1976 in Zürich stattfand. Ab diesem Zeitpunkt richtete die Gesellschaft praktisch alljährlich einen Kongress aus, die Zahl der Teilnehmenden stieg, die Dauer verlängerte sich und die Partner wechselten je nach Thema.

\section{Identitätsbildung, Zusammenhalt, Unterstützung der Forschenden}

Am Kongress von 1976 wurde das Programm der neuen Gesellschaft nochmals bekräftigt (SGBF, Protokoll der Generalversammlung vom 25. Juni 1976). In den folgenden Jahren kam gleichsam ein Punkt nach dem andern zur Umsetzung. So erschien 1977 das erste Bulletin zum Informationsaustausch innerhalb der Gesellschaft und nach aussen. Ebenfalls 1977 wurden die Grundlagen gelegt für eine Publikationsreihe mit Beiträgen zur Bildungsforschung im PeterLang-Verlag. Unter dem Titel «Explorationen. Studien zur Erziehungswissenschaft» bzw. «Exploration. Collection de la Société Suisse pour la Recherche en Education» sorgte je eine Equipe in der deutsch- und der französischsprachigen Schweiz für die Herausgabe der Werke. Bis heute sind in dieser Reihe rund 200 Bücher erschienen.

1979 wurde die Zeitschrift gegründet. Im Editorial der ersten Nummer der in deutscher und französischer Sprache publizierten Zeitschrift werden zwei Ziele hervorgehoben: Als Fachorgan wollte die Zeitschrift der Kommunikation unter Bildungswissenschaftern dienen, aufgrund der Praxisnähe der Bildungsforschung aber auch ein Ort des Gesprächs und der gedanklichen Auseinandersetzung zwischen Wissenschaft und Praxis im Bildungswesen sein (Hedinger, 1979). Diese Ziele spiegeln sich im Titel der Zeitschrift, der bis 1999 unverändert blieb: «Bildungsforschung und Bildungspraxis / Education et recherche».

Gleichzeitig bemühten sich Vorstand und forschungspolitische Kommission erfolgreich um ein Nationales Forschungsprogramm [NFP]. Im Rahmen 
des NFP 10, dessen inhaltliche Ausrichtung «Education et vie active» schon am SGBF-Kongress von 1977 in Genf diskutiert worden war, nahmen die Forschenden 1980 ihre Arbeit auf (Steiner, 1980). Neben den Arbeitsgruppen, und der Zeitschrift boten auch die jährlichen Kongresse eine wichtige Plattform für den wissenschaftlichen Austausch. Sie lassen sich inhaltlich folgenden Themenkreisen zuordnen:

- Bildung und Arbeit (1977, 1998, 2001)

- Wissen, Lernen, Unterricht (1994, 1996, 2006, 2009, 2013, 2014)

- Bildungsinstitutionen und -stufen (1979, 1996, 2006, 2009, 2013, 2014)

- Bildungsqualität, -wirksamkeit und -ungleichheit (1991, 2002, 2007, 2015)

- Bildungssystem (1978, 1992, 1993, 1999, 2004, 2011)

- Bildungsforschung, Erziehungswissenschaften (1983, 1985, 1987, 1995, 2000, 2008, 2010).

Auf zwei Kongresse soll speziell hingewiesen werden: zum einen auf den Jubiläumskongress zum 10-jährigen Bestehen der SGBF und zum andern auf den 25. Jahreskongress zum Thema Bildungswissenschaften. 1985 trafen sich die SGBF-Mitglieder in Aarau und blickten auf die ersten 10 Jahre der Existenz ihrer wissenschaftlichen Gesellschaft zurück. Die Jubiläumsreden des Gründungs- und des damals amtierenden Präsidenten zeigen zum einen den Stolz auf das Erreichte: Zeitschrift, Bulletin, Publikationsreihen, Jahreskongresse, Arbeitsgruppen, Nationales Forschungsprogramm hatten realisiert werden können (Goldschmid, 1985). Zum andern betonte vor allem der amtierende Präsident, Fritz Oser, Pädagogik-Professor an der Universität Fribourg, das Identitätsproblem, das die Gesellschaft seiner Auffassung nach nicht hatte lösen können. Zwar würden Vertreterinnen und Vertreter vieler Disziplinen der SGBF beitreten, sich aber zu wenig mit den Zielen der SGBF identifizieren und sich dafür engagieren, weshalb der Gesellschaft die nötige Schlagkraft fehle (Oser, 1985).

Der 25. Jahreskongress der SGBF im Jahr 2000 in Genf rückte die «Bildungswissenschaften" ins Zentrum. Als Diskussionsgrundlage diente eine Bestandesaufnahme, deren Erstellung vom Schweizerischen Wissenschaftsrat, der CORECHED und der Schweizerischen Koordinationsstelle für Bildungsforschung unterstützt worden war und die nach dem Kongress in eine Prospektivstudie mündete (Hofstetter \& Schneuwly, 2001). Die Studie griff die teilweise schwierigen Rahmenbedingungen und daraus resultierenden Qualitätsprobleme auf (z.B. institutionelle Fragmentierung, Mangel an langfristig gesicherten Forschungsstellen, inhaltliche Konzentration auf bereits gut erforschte Bereiche, Ungleichgewicht zwischen Grundlagen- und anwendungsorientierter Forschung) und formulierte Entwicklungsperspektiven. Ebenfalls zur Diskussion stand die Neuausrichtung und verstärkte Wissenschaftsorientierung der Zeitschrift, verbunden mit der Umbenennung in «Schweizerische Zeitschrift für Bildungswissenschaften / Rivista svizzera di scienze dell'educazione / Revue suisse des sciences de l'éducation» ${ }^{4}$ (SGBF, 2000b). 
Nach dem Kongress 2000 wurde die Gesellschaft reorganisiert und die Statuten entsprechend angepasst. Neu werden im Zweckartikel Arbeitsgruppen explizit als Ort der Zusammenarbeit genannt, die Informationsvermittlung an die Mitglieder als Aufgabe definiert und die Nachwuchsförderung zu einem zentralen Anliegen erklärt.

Bilanzierend sei festgehalten, dass die Schweizerische Gesellschaft für Bildungsforschung in den erste Jahren ihres Bestehens mit den Arbeitsgruppen, dem Bulletin, der Zeitschrift und den Kongressen alle Voraussetzungen geschaffen hat, um den Forschenden Gefässe für den wissenschaftlichen Austausch zur Verfügung zu stellen. Die SGBF spielte im Prozess der Konstituierung des Forschungsbereiches und der Forschergemeinschaft als "pluridisziplinäre Disziplin» (Hofstetter \& Schneuwly 2001, S. 31) eine wichtige Rolle. Während zu Beginn die disziplinäre Offenheit und ein starker Praxisbezug der Bildungsforschung betont und auch im Namen der Gesellschaft sowie der Zeitschrift zum Ausdruck gebracht wurden, schärften sich um die Jahrtausendwende Profil und Anspruch. Mit der aktiven Rolle, welche die SGBF bei der Gründung der European Educational Research Association [EERA] spielte, förderte sie die internationale Öffnung und Vernetzung und trug damit der gesellschaftlichen Entwicklung im Bildungsbereich wie auch den veränderten Ansprüchen an Forschung Rechnung. Der Diskurs um die Qualität von Bildungsforschung mündete nicht zuletzt in die Neuausrichtung der Zeitschrift mit Peer-Review-Verfahren und stärker betonter Mehrsprachigkeit.

\section{Forschungspolitische Aktivitäten}

Nach der Gründung der Gesellschaft hatte die forschungspolitische Kommission, parallel zu den Bemühungen um ein Nationales Forschungsprogramm, auch die Arbeit an einem Entwicklungsplan für die Bildungsforschung aufgenommen. Am Jubiläumskongress zum 10-jährigen Bestehen der SGBF von 1985 wurde der Entwicklungsplan intensiv debattiert. Neben Diskussionsgruppen unter Mitgliedern der Gesellschaft, nahmen auch eine international zusammengesetzte Forschergruppe sowie Vertreter der Erziehungsdirektorenkonferenz und des Bundes dazu Stellung (SGBF, Programm des Kongresses 1985). Nach einer Phase der Überarbeitung wurde der Entwicklungsplan 1988 als Sondernummer der Zeitschrift «Bildungsforschung und Bildungspraxis» veröffentlicht (SGBF, 1988). Er umfasst 16 Empfehlungen, die in folgende vier Pakete gruppiert sind:

1. Stärkung von Rolle und Stellung der Bildungsforschung

2. Stärkung der Forschungsstruktur

3. Forschungskompetenz und Nachwuchsförderung

4. Finanzierung von Bildungsforschung.

Als ein wichtiges Instrument zur Umsetzung dieser Forderungen galten die Nationalen Forschungsprogramme. 1990 konnten die Vorbereitungen für ein 
zweites NFP im Bildungsbereich in Angriff genommen werden. Es war dem Thema «Wirksamkeit unserer Bildungssysteme» gewidmet, zu dem auch der SGBF-Kongress 1991 in Genf durchgeführt wurde (Matthey, 1990). Inhaltlich klinkte sich die SGBF mit dem Programm ein in den öffentlichen und bildungspolitischen Diskurs um Fragen der Qualität und der Wirksamkeit von Bildungssystemen und Bildungspolitiken (SNF, 1991). Laut Ausführungsplan sollten die Forschungsarbeiten aus der Sicht der formativen Evaluation von Bildungssystemen konzipiert werden (ebd.). Neben den inhaltlichen Zielen des Programms war eines auch auf die Vergrösserung des Potentials der Bildungsforschung ausgerichtet. Nach fast 10 Jahren Dauer fand das NFP 33 anlässlich des SGBF-Jahreskongresses im Oktober 1999 in Neuenburg seinen Abschluss. Die Evaluation zeigte, dass das Forschungsprogramm zwar einen kurzfristigen Investitionsschub ausgelöst hatte, ohne allerdings nachhaltig für längerfristige Bildungsforschungskapazitäten sorgen zu können (Meyer, 1999). Analysen der Projektdatenbank der Schweizerischen Koordinationsstelle für Bildungsforschung weisen sogar auf einen finanziellen Kompensationseffekt hin, in dem Sinne, dass in der Laufzeit des NFP 33 andere Finanzierungsquellen spärlicher flossen (Grossenbacher \& Oggenfuss, 2015a).

Ebenfalls zu Beginn der 1990er Jahre beteiligte sich die SGBF an der vom Wissenschaftsrat vorgeschlagenen «Untersuchung zur Situation der Sozialwissenschaften in der Schweiz» (SSRE, Procès-verbal de la séance du Conseil du 20 février 1991). Die Untersuchung zielte darauf ab, die Sozialwissenschaften nicht zuletzt im Kampf um knappe Forschungsgelder zu stärken. Zwar profitierte die Bildungsforschung von den Ergebnissen des SOWI-Prozesses wie beispielsweise der Lancierung des Schwerpunktprogramms "Zukunft Schweiz», der Einsetzung eines wissenschaftspolitischen Rats der Sozialwissenschaften oder der Errichtung des Daten-Archivs SIDOS, das mittlerweile ins Schweizer Kompetenzzentrum Sozialwissenschaften FORS übergegangen ist. Die Bemühungen der SGBF allerdings, im Rahmen der ab 2001 neu eingerichteten millionenschweren Nationalen Forschungsschwerpunkte NFS auch einen für die Bildungsforschung zu errichten, blieben erfolglos.

In die Präsidentschaftszeit von Bernard Schneuwly, Erziehungswissenschafter an der Universität Genf, fallen mehrere forschungspolitische Stellungnahmen. Im März 1997 verabschiedete der Vorstand «Empfehlungen zu Forschung und Entwicklung an Pädagogischen Hochschulen» (SGBF, 1997). Die absehbaren Veränderungen in der Bildungsforschungslandschaft gaben Anlass, auf die bestehenden Probleme hinzuweisen und zu versuchen, eine Verschärfung durch weitere Zersplitterung sowie personelle und finanzielle Unterdotierung der Bildungsforschung zu verhindern. Die SGBF plädierte für die Einrichtung potenter Forschungszentren an den Pädagogischen Hochschulen und für eine intensive Kooperation von Pädagogischen Hochschulen und den bestehenden Institutionen der Bildungsforschung. Die SGBF empfahl auch, die Qualität von Forschung und Entwicklung an Pädagogischen Hochschulen zu sichern. Als 
zentrale Elemente der Qualitätssicherung nannte die SGBF die Teilnahme an nationalen und internationalen Diskussionsnetzen sowie die Veröffentlichung der Resultate (ebd.).

In Reaktion auf eine EDK-Studie zur Berufsbildungsforschung (Kiener, 1999) und entsprechende Thesen des Bundesamts für Berufsbildung und Technologie $[\mathrm{BBT}]$ bzw. der Kommission für Technologie und Innovation KTI (BBT, 2000) verabschiedete die SGBF im Sommer 2000 Grundsätze und Prioritäten zur Berufsbildungsforschung (SGBF, 2000a). Im Prinzip befürwortete die Gesellschaft den Aufbau von Netzwerken und Kompetenzzentren für Berufsbildungsforschung, wollte jedoch verhindern, dass diese sich gleichsam ausserhalb der allgemeinen Bildungsforschung und deren Strukturen und Themen konstituierten. Sie sprach sich auch für die Priorisierung von pädagogischen und bildungssoziologischen Themen aus (Qualifikationen, Übergänge und Chancengleichheit) und erklärte ökonomische Fragen nach Erträgen und Effizienz zwar für wichtig aber nachrangig (ebd.).

Auch die am 25. Jahreskongress der SGBF im Jahr 2000 diskutierte Studie zu Stand und Perspektiven der Bildungswissenschaften (Hofstetter \& Schneuwly, 2001) reflektierte zwar vor allem Arbeitsbedingungen, Personalsituation und Produktion der universitären erziehungswissenschaftlichen Institute, bezog jedoch auch den forschungspolitischen Rahmen in die Betrachtungen mit ein.

Alle im Lauf der Jahre gesammelten Informationen zur Bildungsforschung in der Schweiz bildeten das Material für den Grundlagenbericht, den die CORECHED für eine externe Beurteilung der Bildungsforschung in der Schweiz durch das CERI der OECD zusammenstellte (CORECHED, 2006). Diese Review mit Beteiligung internationaler Experten, die in der Präsidialzeit von Stefan Wolter, Bildungsökonom und Direktor der Koordinationsstelle für Bildungsforschung, stattfand, fiel kritisch aus. Die Expertengruppe stellte fest, dass die Bildungsforschung in der Schweiz ihr Potential nicht ausschöpfe und sie forderte dazu auf, die politische und praktische Nutzung der Forschung zu fördern, sowie dem Capacity-Building, der Qualitätssicherung und der Internationalisierung mehr Aufmerksamkeit zu schenken (OECD/CERI, 2007). Der Bericht löste in der Community kritische Reaktionen aus, die insbesondere die fehlende Berücksichtigung neuester Entwicklungen des Forschungsfeldes im Grundlagenbericht sowie die auf die unmittelbare Nutzbarkeit der Forschung für Praxis und Politik fokussierende Analyseperspektive bemängelten. Der Bericht wurde auf einer eigens dafür von der SGBF organisierten Tagung intensiv diskutiert (Grossenbacher, 2008).

In den Jahren 2008 bis 2011 beteiligte sich die SGBF unter Federführung von Matthis Behrens, damaliger SGBF-Präsident und Direktor des Institut de recherche et de documentation pédagogique [IRDP], an einem Europäischen Projekt mit Namen EERQI (European Educational Research Quality Indicators). Das Projekt (initiiert von der EERA) zielte darauf ab, die weltweite Bedeutung und Wettbewerbsfähigkeit der europäischen Bildungsforschung 
zu stärken und dem Übergewicht der auf den englischen Sprachraum ausgerichteten Qualitätsevaluationsinstrumente eine Alternative entgegenzusetzen (Gogolin, Aström \& Hansen, 2014).

Zusammenfassend sei festgehalten, dass die SGBF sich von Anfang an bemühte, das Forschungsfeld genau zu beobachten, den Stellenwert der Bildungsforschung gegenüber Politik, Verwaltung und Praxis zu stärken, die Finanzierungslage zu verbessern, die Rahmenbedingungen für die Forschung in diesem Bereich zu optimieren. Sie setzte sich auch immer wieder selbstkritisch mit den Funktionsmechanismen des eigenen Wissenschaftsbereiches, seinen Strukturen, Prozessen und Produktionen auseinander und griff ein, wenn sie die Rahmenbedingungen einer qualitätsvollen Arbeitsweise bedroht sah. Schliesslich beteiligte sie sich auch an den europaweiten Reflexionen zum Stellenwert der Bildungswissenschaften. Mit ihren Bestandesaufnahmen und den forschungspolitischen Stellungnahmen trug sie wesentlich zum selbstkritischen Diskurs über Qualität in der Bildungsforschung bei.

\section{Kompetenzaufbau und Nachwuchsförderung}

Bereits früh hatte die Gesellschaft erste Bestrebungen zur Weiterbildung von Forschenden aufgenommen. So wurde in Erfüllung der Empfehlung 4 des Entwicklungsplans, ein Projekt für eine Centrale suisse pour la formation continue des chercheurs en éducation vorgelegt. Da in der Westschweiz bereits Bestrebungen der Universitäten für eine Graduiertenausbildung in Gang waren, startete man in der Deutschschweiz mit einem Pilotversuch «Fortbildung à la carte» (Forum für Forschung und Entwicklung im Bildungsbereich, 1989). In den 1990er Jahren wurden die Fortbildungsaktivitäten in die Arbeitsgruppen bzw. die Rahmenaktivitäten des NFP 33 verlegt. Mit dem Aufbau von Summer Schools in den Sozialwissenschaften und von zahlreichen universitären Weiterbildungsangeboten verlagerte sich der Schwerpunkt der SGBF von der Fortbildung auf die Nachwuchsförderung. Vorgängig zum Jahres-Kongress 2007 wurde eine Vorkonferenz für den wissenschaftlichen Nachwuchs organisiert und seither ist dieses Angebot beibehalten worden. Die Themen der Konferenzen kreisen um Fragen, die den Nachwuchs sehr praktisch interessieren (methodische Verfahren, Drittmittelbeschaffung, Publikationsstrategien, wissenschaftliche Karriereplanung, Doktoratsprogramme etc.).

Im Jahr 2012 lancierte die erste Präsidentin der SGBF, Katharina Maag Merki, zudem einen Nachwuchsförderpreis, der - im Zweijahresrhythmus verliehen - Arbeiten junger Forschender auszeichnet.

Zusammenfassend sei festgehalten, dass Kompetenzaufbau im eigenen Forschungsgebiet stets ein wichtiges Anliegen der Gesellschaft war, wobei sich der Schwerpunkt von der Fortbildung zur Nachwuchsförderung verlagert hat. Unter der ersten weiblichen Vorsitzenden hat sie ihre spezifische Gestalt 
angenommen, die auch von ihrem Nachfolger, Roland Reichenbach, und der amtierenden Präsidentin, Isabelle Dominique Mili, gepflegt wurde und wird. Die Vorkonferenzen mit ihren realen Angeboten und der Nachwuchsförderpreis mit seinem symbolischen Charakter zeugen davon. Weitere Möglichkeiten bieten die aktive Mitarbeit in einer Arbeitsgruppe der SGBF oder die Zeitschrift.

\section{Die Zeitschrift der SGBF - eine Analyse mit explora- tivem Charakter}

Der Zeitschrift der SGBF ist der letzte Teil dieses Beitrages gewidmet. Bereits zum 30-jährigen Bestehen der 1979 gegründeten Zeitschrift haben Lucien Criblez und Karin Manz eine Analyse vorgelegt (Criblez \& Manz, 2008). Die in diesem umfassenden Überblick dargestellten Informationen sollen hier nicht wiederholt, jedoch teilweise als Bezugspunkte genutzt werden. Das im Folgenden Präsentierte basiert auf einer Durchsicht ${ }^{5}$ der Jahrgänge 2000 bis 2014 und damit der zu Beginn des Jahrtausends neu konzipierten «Schweizerischen Zeitschrift für Bildungswissenschaften» [SZBW].

Als wissenschaftliche Zeitschrift konzipiert, erscheint die SZBW drei Mal jährlich, die Ausgaben sind zumeist als Themennummern gestaltet, ergänzt mit einem oder zwei allgemeinen Beiträgen. Die Artikel werden nach einem Review-Verfahren aufgenommen, sie erscheinen in französischer, deutscher, englischer oder italienischer Sprache, mit Abstracts in den jeweils anderen Sprachen. Für die folgenden Zusammenstellungen wurden die Editorials und die Rezensionen nicht mit einbezogen.

- Zwischen 2000 und 2014 hielten sich die französischsprachigen und die deutschsprachigen Beiträge in etwa die Waage mit 45\% bzw. 44\%. Englischsprachige Artikel waren mit 8\%, italienischsprachige mit 3\% vertreten. Vergleicht man dieses Ergebnis mit der erwähnten Analyse von Criblez und Manz (2008), zeigt sich, dass die Zeitschrift sich weiter geöffnet hat sowohl gegenüber dem englischen wie dem italienischen Sprachraum.

- Gemäss Criblez und Manz (2008) hat die Zeitschrift mit der Neukonzeption im Jahr 2000 an Umfang stark zugelegt. Seit 2000 beträgt der Umfang stabil 500 bis 600 Seiten pro Jahrgang, alle Beiträge (auch Editorials, Rezensionen und Biografien) mit eingerechnet.

- In ihrer Analyse weisen Criblez und Manz (2008) auf den steigenden Frauenanteil unter den Beitragenden hin. Über die von ihnen betrachteten 29 Jahrgänge (1979-2007) berechnet, betrug er 28\%. Werden nur die 15 Jahrgänge seit 2000 berücksichtigt, beträgt der Frauenanteil 47\%. Über die 60\%-Marke klettert dieser Anteil dann, wenn gewisse Themen wie Genderfragen, Heterogenität, Interkulturalität sowie Literalität oder Sprachvermittlung im Zentrum stehen. Und auch in diesem jüngeren Zeitraum gibt es noch Jahrgänge, in denen der Frauenanteil weniger als 30\% beträgt.

- Die Autorinnen und Autoren der Zeitschrift haben ihren Arbeitsort in der Regel mehrheitlich in der Schweiz, häufig aber auch in Deutschland, Frank- 
reich oder Kanada. Seltener sind Autorinnen oder Autoren aus den Nachbarländern Österreich und Italien vertreten.

- Die Analyse von Criblez und Manz (2008) zeigte über die gesamte Erscheinungszeit von 1979 bis 2007 einen Anteil von Einzelautorschaften von 72\%, gegenüber $18 \%$ Doppelautorschaften und nur 10\% Autorenteams mit drei und mehr Autor/innen. Betrachtet man nur die Jahrgänge seit 2000, ändert sich das Bild. Der Anteil der Einzelbeiträge sank auf 57\%, jener der Doppelautorschaften wuchs auf $22 \%$ und die Beiträge von Autorenteams verdoppelten sich auf 21\%. Der von Criblez und Manz (2008) noch als stabil bezeichnete Überhang der Einzelautorschaft reduzierte sich ab 2009, die Beiträge von Teams nahmen stark zu (Abb. 1).

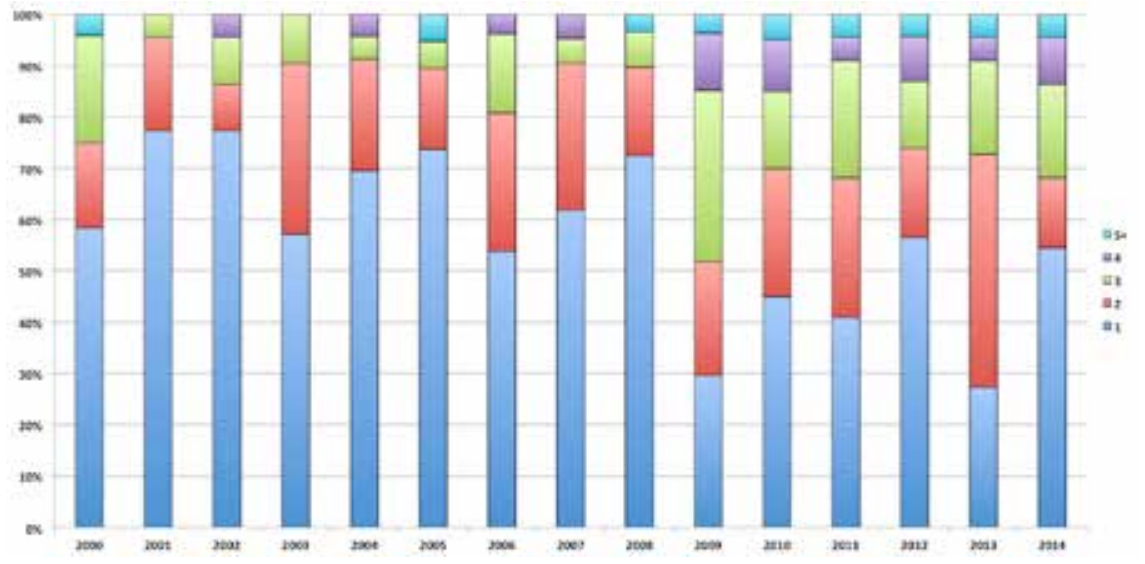

Abbildung 1: Schweizerische Zeitschrift für Bildungswissenschaften, Jahrgänge 2000-2014, nach Anzahl der Autorinnen bzw. Autoren pro Beitrag

- Unterscheidet man bei den Jahrgängen seit 2000 die Beiträge grob nach ihrem Zugang zum Thema und teilt sie in Anlehnung an Zierer, Ertl, Phillips und Tippelt (2013) den Kategorien «empirisch» (Generierung und Interpretation neuer Daten oder Neuauswertung bestehender Daten) und «nicht-empirisch» (Verarbeitung bestehender Daten oder Texte) $\mathrm{zu}^{6}$, wird deutlich, dass die Beiträge mehrheitlich empirisch orientiert sind, vor allem ab 2010 (Abb. 2).

- Die Analyse der Jahrgänge 2000 bis 2014 ergibt einen grossen Anteil von Beiträgen, bei denen kein klarer Stufenbezug feststellbar ist (32\%). Weitere $22 \%$ der Beiträge beziehen sich auf die Volksschule, $18 \%$ betreffen mehrere Stufen, bearbeiten also beispielsweise Übergangsthemen. Untervertreten sind der Elementarbereich (5\%), die Sekundarstufe II (9\%) sowie die Weiterbildung (3\%). Seit 2009 zugelegt hat der Anteil an Beiträgen mit Bezug zur Tertiärstufe. 


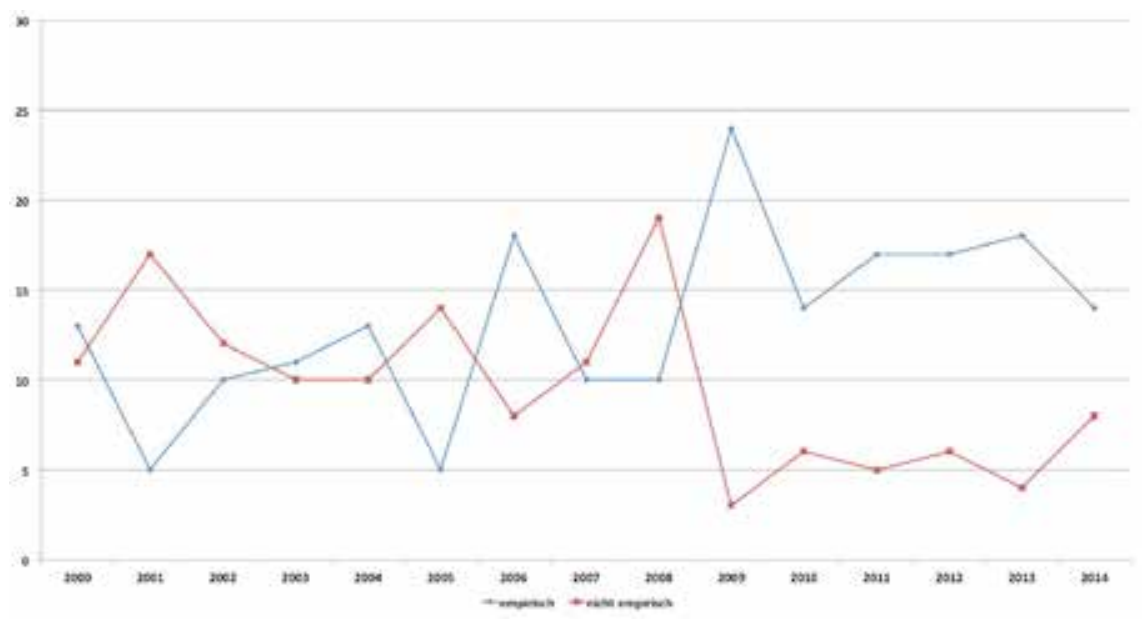

Abbildung 2: Schweizerische Zeitschrift für Bildungswissenschaften, Jahrgänge 2000-2014, nach wissenschaftlichem Zugang der Beiträge

Die Übersicht zeigt starke Anzeichen dafür, dass die mit der Neukonzeption der Zeitschrift angestrebten Veränderungen umgesetzt werden konnten. Die Zeitschrift ist wissenschaftlicher geworden und hat sich vermehrt international ausgerichtet. Aber sie kann inhaltliche Einseitigkeiten der wissenschaftlichen Produktion selbstverständlich nicht abfedern. Dass z. B. gewisse Stufen des Bildungssystems von der Forschung vernachlässigt wurden, zeigen auch die bis heute wiederholt vorgelegten Analysen, die im Beitrag Erwähnung finden.

\section{Zusammenfassung}

Einführend wurde festgehalten, dass die systematische Aufarbeitung der Geschichte der SGBF eine noch offene Aufgabe sei. Die SGBF, ihre Organe, ihre Aktivmitglieder in den Arbeitsgruppen und selbstverständlich die Präsidenten und Präsidentinnen waren und sind stets bemüht, die Gesellschaft zukunftstauglich zu machen, wofür ihnen Dank auszusprechen ist. Dieser Beitrag konnte nur einige Linien zu wenigen Aspekten der zurückliegenden vielfältigen Aktivitäten nachzeichnen. Dabei kam nicht nur die historische Systematik zu kurz; unerwähnt bleiben mussten auch wichtige Aspekte wie die Entwicklung der Institutionen, personale und institutionelle Vernetzungen, internationale Beziehungen oder Zusammenarbeitsstrukturen innerhalb der Schweizerischen Akademien. Nur in Ansätzen konnte gezeigt werden, dass den Aktivitäten der Gesellschaft seit ihrem Bestehen ein lebendiger Diskurs um Bildungs- und Forschungsqualität zugrunde liegt. Diesen expliziter herauszuarbeiten bleibt ebenfalls ein offenes Forschungsdesiderat. 


\section{Anmerkungen}

1 Der Beitrag basiert auf einer Keynote am Kongress der SGBF vom 26.9.-1.7.2015 in St. Gallen.

2 Die Auswahl der dargestellten Aspekte erfolgte mit der Absicht, eine Verbindung zum Thema des SGBF-Kongresses 2015 (Qualitäts- und Bildungsdiskurs) herstellen zu können. Als Quellen dienten Protokolle von Gründungsversammlung, Vorstandssitzungen und Generalversammlungen sowie das interne Bulletin der SGBF, das zwischen 1977 und 2001 in gedruckter Form erschien. Diese Unterlagen entstammen dem Archiv der SGBF, das sich in den Räumlichkeiten der Schweizerischen Koordinationsstelle für Bildungsforschung SKBF in Aarau befindet.

3 Die zitierten Bestandesaufnahmen beruhen zum grossen Teil auf Auswertungen der Projektdatenbank der SKBF. Gemäss ihrem Auftrag dokumentiert die SKBF in dieser Datenbank Projekte der schweizerischen Bildungsforschung oder internationaler Bildungsforschung mit einem Bezug zur Schweiz. Die Projekte werden aufgrund eigener Suchbewegungen der SKBF erfasst oder von den Forschenden selbst gemeldet. Während zu Beginn der Projekterfassung teilweise auch Informationen zu Entwicklungsprojekten sowie zu laufenden Forschungsprojekten einflossen, wurden die Bedingungen für einen Neueintrag ab 2000 rigoroser gestaltet. Aufgenommen werden abgeschlossene Forschungsprojekte, aus denen erste Ergebnisse verfügbar sind und zu denen mindestens eine öffentlich zugängliche Publikation vorliegt.

4 Der vollständige Titel der Zeitschrift manifestiert die Bereitschaft der Zeitschrift, die mehrsprachige Bildungsforschungsproduktion in der Schweiz abzubilden. Auch englischsprachige Artikel finden Eingang und jedem Artikel ist eine Zusammenfassung in den vier Sprachen (Deutsch, Englisch, Französisch, Italienisch) beigefügt.

5 Der Zugang ist als explorativ zu bezeichnen, die Zuordnungen wurden nicht überprüft.

6 Der Kategorie «nicht-empirisch» zugeordnet wurden theoretisch und forschungsmethodisch orientierte Beiträge, Forschungsüberblicke, Literaturanalysen sowie Beiträge, in denen bereits anderweitig publizierte Daten referiert werden. Zierer et al. (2013) weisen darauf hin, dass diese begriffliche Unterscheidung nicht unproblematisch sei, da auch hermeneutische, phänomenologische und dialektische Verfahren auf Erfahrung im weitesten Sinne zurückgriffen (ebd., 412).

\section{Literatur}

Bain, D., Brun, J., Hexel, D. \& Weiss, J. (Éd.). (2001). L'épopée des centres de recherche en éducation en Suisse 1960 - 2000. Die Geschichte der Bildungsforschungsstellen in der Schweiz 1960 - 2000. Neuchâtel: IRDP.

BBT [Bundesamt für Berufsbildung und Technologie]. (Hrsg.) (2000). Thesen zur Berufsbildungsforschung und Organisation des Leistungsbereichs. Bericht der Projektgruppe applikationsorientierte Berufsbildungsforschung des BBT. Bern: BBT.

BBT [Bundesamt für Berufsbildung und Technologie] (Hrsg.). (2007). Berufsbildungsforschung Schweiz: das BBT-Förderprogramm. Bern: BBT. Zugriff am 22.3.2016 unter http:// www.sbfi.admin.ch/berufsbildung/01528/index.html?lang=de

CORECHED [Schweizerische Konferenz Bildungsforschung]. (1996). Erste Berichterstattung über Stand, Entwicklung und Tendenzen der Bildungsforschung in der Schweiz sowie deren Beziehungen zu Bildungspolitik, -verwaltung und -praxis. Aarau: SKBF.

CORECHED. (2006). OECD/CERI Review: Educational Research and Development in Switzerland. Country Background Report. Aarau: SKBF. Zugriff am 22.3.2016 unter http://www.sgbf.ch/jahreskongresse/CERI_SWISS-CBR_dec06.pdf

Criblez, L. (2002). Fragil und unstet - Zur Entwicklung der Erziehungswissenschaften an den Universitäten der deutschsprachigen Schweiz. In R. Hofstetter \& B. Schneuwly (Hrsg.), 
Erziehungswissenschaft(en). 19.-20. Jahrhundert. Zwischen Profession und Disziplin (S. 425-453). Bern: Lang.

Criblez, L. \& Manz, K. (2008). Im Dienste der Bildungspraxis oder der Bildungsforschung? Analysen und empirische Befunde zur Zeitschrift der Schweizerischen Gesellschaft für Bildungsforschung (SGBF) anlässlich ihres 30-jährigen Bestehens. Schweizerische Zeitschrift für Bildungswissenschaften, 3, 419-445.

Forum für Forschung und Entwicklung im Bildungsbereich. (1989). Fortbildung von Bildungsforschern in der Schweiz. SGBF Bulletin, 3, 9-11.

Gogolin, I., Aström, F. \& Hansen, A. (Éd.). (2014). Assessing Quality in European Educational Research. Indicators and Approaches. Wiesbaden: Springer.

Goldschmid, M. L. (1985). La SSRE a déjà 10 ans. Bulletin SSRE, 3, 8.

Gretler, A. (2000). Die schweizerische Bildungsforschung der Nachkriegszeit im Spiegel ihrer Institutionen und ihrer Themen - von der Geschichte zu aktuellen Fragestellungen. Schweizerische Zeitschrift für Bildungswissenschaften, 1, 111-144.

Grossenbacher, S. \& Gretler, A. (1992). Untersuchung zur Situation der sozialwissenschaftlichen Forschung in der Schweiz. Bericht der Bildungsforschung (FOP 1/1992). Bern: Schweizerischer Wissenschaftsrat.

Grossenbacher, S. (2008). Tagung "Perspektiven zur Weiterentwicklung der Bildungsforschung in der Schweiz». Bulletin Schweizerische Akademie der Geistes- und Sozialwissenschaften, 3, 63-64.

Grossenbacher, S. \& Oggenfuss, C. (2015a). "Information Bildungsforschung» - 40 Jahre Forschungsdokumentation der SKBF (SKBF Staff Paper 15). Zugriff am 22.3.2016 unter http://skbf-csre.ch/fileadmin/files/pdf/publikationen/Staffpaper15.pdf

Grossenbacher, S. \& Oggenfuss, C. (2015b). Bildungsforschung an Lehrerbildungsinstitutionen: Ein Auszug aus der SKBF-Datenbank. Beiträge zur Lehrerinnen- und Lehrerbildung, 3, 402-415.

Hedinger, U. K. (1979). Editorial. Bildungsforschung und Bildungspraxis, 1, 3-4.

Hofstetter, R. \& Schneuwly, B. (2001). Les Sciences de l'éducation en Suisse. Evolution et prospectives. Bern: Center d'études de la science et de la technologie.

Huberman, M. (1989). Situationsanalyse der pädagogischen Abteilungen der Kantone in der Deutschschweiz. Bildungsforschung und Bildungspraxis, 3, 66-82.

Kiener, U. (1999). Berufsbildungsforschung in der Schweiz: Grundlinien eines Konzeptes. Expertenbericht (EDK-Dossier 58A). Bern: EDK.

Mathey, M. (1990). Editorial. SGBF Bulletin, 3, 5.

Meyer, T. (1999). Evaluative Befragung der NFP-33-Forscherinnen und -Forscher. SGBF Bulletin, 2, 43-46.

OECD/CERI. (2007). National Review of Educational R\&D: Switzerland. Zugriff am 22.3.2016 unter http://www.coreched.ch/publikationen/experts_report_en.pdf

Oser, F. (1985). 10 Jahre Schweizerische Gesellschaft für Bildungsforschung. SGBF Bulletin, 3, 5-7.

Patry, J.-L. \& Gretler, A. (1992). Bildungsforschung in der Schweiz 1970 bis 1990: Interdisziplinarität und Forschung-Praxis-Bezug. Empirische Pädagogik, 1, 33-77.

SGBF [Schweizerische Gesellschaft für Bildungsforschung]. (1988). Entwicklungsplan der schweizerischen Bildungsforschung. Bildungsforschung und Bildungspraxis (Sondernummer). Aarau: SKBF.

SGBF Bulletin / Bulletin SSRE, Jahrgänge 1977-2001.

SGBF. (1997). Empfehlungen zu Forschung und Entwicklung an Pädagogischen Hochschulen, verabschiedet vom SGBF-Vorstand am 13. März 1997. SGBF Bulletin, 2, 6-50.

SGBF. (2000a). Forschung in der Berufsbildung - Grundsätze und Prioritäten. Positionen der Schweizerischen Gesellschaft für Bildungsforschung (vom 6. Juni 2000). SGBF Bulletin, 2, 32-35. 
SGBF. (2000b). Protokoll der SGBF-Generalversammlung (FPSE, Université de Genève, 21 septembre 2000). SGBF Bulletin, 3, 16-25.

SGBF, Statuten (Stand 2012). Zugriff am 22.3.2016 unter http://www.sgbf.ch/portrait/ statuten/Statuten_SGBF_d.2012.pdf

SNF [Schweizerischer Nationalfonds zur Förderung der wissenschaftlichen Forschung]. (Hrsg.). (1991). Nationales Forschungsprogramm 33. Die Wirksamkeit unserer Bildungssysteme angesichts der demographischen und technologischen Entwicklung und angesichts der Probleme in der mehrsprachigen Schweiz. Ausführungsplan. Bern: SNF.

Steiner, G. (1980). Préface du bulletin eva (Bulletin d'information de la direction du programm). Bern: Fonds national suisse.

SWR [Schweizerischer Wissenschaftsrat]. (Hrsg.). (1970). Ermittlung über die dringlichen Forschungsbedürfnisse in der Schweiz. Bern: Schweizerischer Wissenschaftsrat.

SWR (Hrsg.). (1973). Forschungsbericht, Band 1. Bern: Schweizerischer Wissenschaftsrat.

Weiss, J. (2001). La recherche extra-universitaire en Suisse romande: caractéristiques et évolution. In D. Bain, J. Brun, D. Hexel \& J. Weiss (Éd.), L'épopée des centres de recherche en éducation en Suisse 1960 - 2000. Die Geschichte der Bildungsforschungsstellen in der Schweiz 1960 - 2000 (S. 325-338). Neuchâtel: IRDP.

Wolter, S. C. (2008). Bildungsberichterstattung auf der Basis von Indikatoren - Eine Situationsbestimmung aus der Schweiz. In LISUM, bm:ukk, EDK (Hrsg.), Bildungsmonitoring, Vergleichsstudien und Innovationen - von evidenzbasierter Steuerung zur Praxis (S. 53-70). Berlin: BWV (Berliner Wissenschaftsverlag).

Zierer, K., Ertl, H. Phillips, D. \& Tippelt, R. (2013). Das Publikationsaufkommen der Zeitschrift für Pädagogik im deutsch-englischen Vergleich. Zeitschrift für Pädagogik, 3, 400-424.

\section{Unveröffentlichte Quellen:}

SGBF, Protokoll der Gründungsversammlung vom 28. Juni 1975. [Archiv SKBF, Dossier SGBF-Vorstand 1975].

SGBF, Protokoll der Generalversammlung vom 25. Juni 1976. [Archiv SKBF, Dossier SGBF-Kongress 1976].

SGBF, Programm des Kongresses 1985. [Archiv SKBF, Dossier SGBF-Kongress 1985].

SSRE, Procès-verbal de la séance du Conseil de la SSRE du 20 février 1991 [Archiv SKBF, Dossier SGBF-Vorstand 1991].

Schlagworte: Bildungsforschung, Erziehungswissenschaften, Schweizerische Gesellschaft für Bildungsforschung, Organisationsgeschichte, wissenschaftliche Zeitschriften 


\section{La Société suisse pour la recherche en éducation - retour sur les grands aspects de ses $\mathbf{4 0}$ ans d'histoire}

\section{Résumé}

L'article met en lumière certains aspects des 40 ans d'histoire de la Société suisse pour la recherche en éducation (SSRE), dans le contexte du développement institutionnel et politique de la recherche en éducation. Dès sa fondation en 1975, cette société s'est donné pour mission - non sans faire preuve d'autocritique de faire évoluer ce domaine de recherche pluridisciplinaire. L'article donne un aperçu des orientations prises par la Société en vue d'affirmer l'identité de ce domaine scientifique et de soutenir les chercheurs. Il présente les objectifs sur lesquels elle s'est concentrée au niveau de la politique de recherche et la manière dont elle a renforcé le développement des compétences et l'encouragement de la relève. Il rend de plus hommage au forum de discussion de la Société, la "Revue suisse des sciences de l'éducation».

Mots-clés: recherche en éducation, sciences de l'éducation, Société suisse pour la recherche en éducation, histoire de l'organisation, revues scientifiques

\section{La Società svizzera di ricerca in educazione: riflessione su quarant'anni di storia}

Riassunto

L'articolo ripercorre alcuni aspetti che hanno caratterizzato i 40 anni di storia della Società svizzera di ricerca in educazione (SSRE), nel contesto dello sviluppo politico e istituzionale della ricerca in educazione. Dalla sua fondazione nel 1975 la Società si adoperata, non senza autocritica, nello sviluppo di questo ambito di ricerca multidisciplinare. Il contributo delinea in modo sintetico le attività intraprese dalla Società per promuovere l'affermazione di questo settore scientifico e il sostegno ai ricercatori, gli obiettivi perseguiti a livello di politiche della ricerca e le modalità con cui ha contribuito allo sviluppo delle competenze incoraggiando le nuove leve. Infine l'articolo rende omaggio al forum di discussione della Società, la «Rivista Svizzera di Scienze dell'Educazione».

Parole chiave: Ricerca in educazione, scienze dell'educazione, Società svizzera di ricerca in educazione, storia dell'organizzazione, riviste scientifiche 


\section{Swiss Society for Research in Education - looking back at its 40-year history}

\section{Summary}

Against the background of the institutional and research policy developments in the field of educational research, this article highlights aspects of the 40-year history of the Swiss Society for Research in Education (SSRE). Since it was founded in 1975, the Society has striven - not without a certain degree of self-criticism - to develop this multidisciplinary research area. The article outlines what the Society has done to raise the profile of this research area and to support researchers, the research policy priorities it has pursued, and how it has encouraged competence building and the promotion of young researchers. Finally, it pays tribute to the Society's discussion forum, the «Schweizerische Zeitschrift für Bildungswissenschaften».

Keywords: Educational research, educational sciences, Swiss Society for Research in Education, organisation history, academic journals 